\title{
Design of power management in Energy Harvesting Devices
}

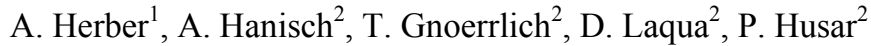 \\ ${ }^{1}$ Technical University of Ilmenau, Department of Biomedical Engineering, Ilmenau, Germany, Adrian.Herber@TU- \\ Ilmenau.de \\ ${ }^{2}$ Technical University of Ilmenau, Department of Biomedical Engineering, Ilmenau, Germany
}

\begin{abstract}
Energy harvesting is an increasingly important capability for a lot of applications. It can be used in biomedical implants but also in other embedded systems. An energy harvesting device needs a functional and efficient power management. Because of the low energy yield it is important to store the harvested energy until enough power is available to accomplish a task like sending data outside the human body. Therefore Li-ion batteries and capacitors are used in this work. Furthermore a typical arrangement of an efficient energy harvesting circuit is discussed and tested.
\end{abstract}

\section{Introduction}

Power management in Energy Harvesting Devices for use in human bodies needs to meet ambitious requirements. Thus long term stability is as important as energy selfsufficiency and implantability of applied biosensors. Energy harvesting manifests several non-idealities, such as power fragmentation, inefficiency of energy storage elements and unpredictability [1].

\subsection{Component requirements}

As a result of smaller components and lower energy consumption, particular attention is paid to microprocessors and A/D converters. Due to more efficient DC/DC converters and a higher energy density of lithium ion batteries named aims can be achieved. The operating conditions of the harvester and its state are essential criteria for the power management [1].

\subsection{Power management}

Nevertheless it is essential to store energy on account of the low power delivered by the energy harvester. Once the available energy is sufficient, the desired task can be accomplished. Furthermore it will be illustrated how harvesting aware power management can be implemented. Also its improvements concerning conventional battery-based power management will be revealed.

To reduce the consumption of energy, as many components of the device as possible should be turned off if not needed. The mean energy consumption of each component then decreases to:

$(\mathrm{P}-$ Power of the device, $\mathrm{t}$ - time $)$

$$
\mathrm{P}_{\text {mean }}=\mathrm{P} \frac{\mathrm{t}_{\text {on }}}{\mathrm{t}_{\text {on }}+\mathrm{t}_{\text {off }}}
$$

\subsection{Determination of state of charge}

It's important that the devices which need the most power only get activated if the energy storage is fully charged or when enough energy is available. To get the state of charge (SOC) of a battery, different methods are available [2].
The method of Coulomb-Counting is among the most accurate procedures to estimate the SOC. Measuring the charge flow directly is the basic concept, so the remaining capacity in a battery can be accumulated over time. Therefore the charging and discharging current need to be quantified [3].

$$
\mathrm{Q}(\mathrm{t})=\mathrm{Q}\left(\mathrm{t}_{0}\right)+\int_{\mathrm{t}_{0}}^{\mathrm{t}} \mathrm{I}(\mathrm{t}) \mathrm{dt}
$$

Usually the current is measured as a voltage drop over a highly precise and very low-ohmic resistor, also known as current shunt. The down side to this method is the relatively high power consumption of the device.

Also the approach through measuring the voltage of the battery and comparing it to the charge/dischargecharacteristics can be used for detecting the SOC. The cell characteristics are provided by the producers, so they can be implemented as a look up table in a microprocessor (MCU) [4].

\section{$2 \quad$ Materials and Methods}

In order to actuate the power management system the ultra-low power MCU MSP430 G2553 (Texas Instruments) supplied by the boost converter and battery charger BQ25504 (Texas Instruments) is used in this work. For testing purposes Li-ion batteries from the Cymbet Corporation and Seiko Instruments were applied (see 2.3). The types of batteries have different charge voltages, so all threshold voltages need to be changed dynamically.

The SOC estimation in our device is realized by comparing the voltage of the battery with a threshold voltage. If the battery voltage exceeds this mark, it is assumed that enough energy is available for the most power consuming task.

We use a voltage controlled oscillator (VCO) to simulate a power consumer. Via the VCO UART (Universal Asynchronous Receiver Transmitter) bits are sent wirelessly using frequency modulation. 4 bytes ( 44 bit including parity, start and stop bit) are sent together when the battery is fully charged. The Spectrum Analyzer E7401A (Hewlett Packard) is used as receiver. 


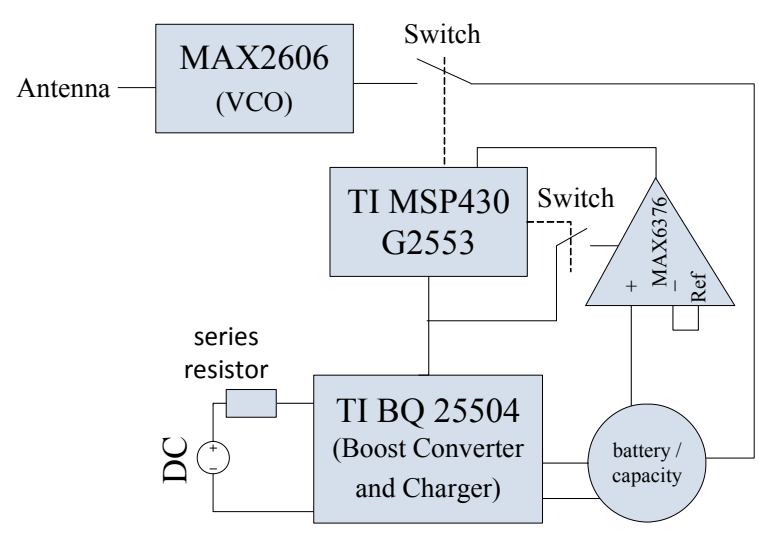

Figure 1: Experimental setup - The switches are MOSFETs.

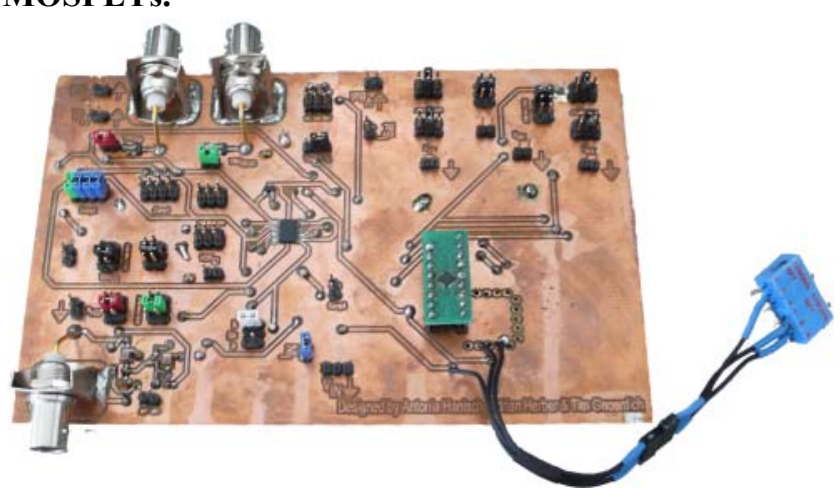

Figure 2: Physical board - The BQ25504 is mounted on the green adapter, the MSP430 G2553 is in the middle of the board

\subsection{Charger \& Protector BQ25504}

The BQ25504 (Texas Instruments) is a tiny boost converter and battery charger for ultra-low power energy harvesting applications $(3.15 \mathrm{~mm} \times 3.15 \mathrm{~mm} \times 1 \mathrm{~mm})$. This device is able to boost low voltages in the range from millivolt $(80 \mathrm{mV})$ to Volt $(3 \mathrm{~V})$. The charge and cutoff voltages are programmable by external resistors [5].

\subsection{Microprocessor MSP430 G2553}

The main function of the ultra-low power Microcontroller MSP430 G2553 (Texas Instruments) is performing periodic tasks like activating the different components and evaluating the results of them. The MCU remains mostly in low power mode 3 which means the CPU and the clocks are disabled, except a very-low-power lowfrequency oscillator $(4-20 \mathrm{kHz})$, which wakes up the CPU in specific periods.

In that mode the MCU consumes about 500 to $700 \mathrm{nA}$ [6]. This device is the only one in the circuit which consumes energy continuously and may not be turned off.

\subsection{Storage elements}

For storing the harvested energy we use batteries from Seiko Instruments and Cymbet Corporation. In addition we use a capacitor (XH311HG) from Seiko Instruments (see Table 1).
Table 1: Available storage elements

\begin{tabular}{|c|c|c|}
\hline Company & Type & Capacity \\
\hline Seiko & MS412FE & $1 \mathrm{mAh}$ \\
\hline Seiko & MS920SE & $11 \mathrm{mAh}$ \\
\hline Cymbet & CBC012 & $12 \mu \mathrm{Ah}$ \\
\hline Cymbet & CBC050 & $50 \mu \mathrm{Ah}$ \\
\hline Seiko & $\mathrm{XH} 311 \mathrm{HG}$ & $20 \mathrm{mF} \approx 6.5 \mu \mathrm{Ah}$ \\
\hline
\end{tabular}

\subsection{Voltage comparison}

The SOC is estimated by a simple comparison operation with a fixed voltage threshold executed by the MCU (see Figure 3). For this comparison a reference element is needed. We use the MAX6376 (Maxim) which is a comparator with a built in factory-trimmed reference voltage [7]. Additionally a voltage divider followed by an operational amplifier is used for changing the threshold voltage level quickly.

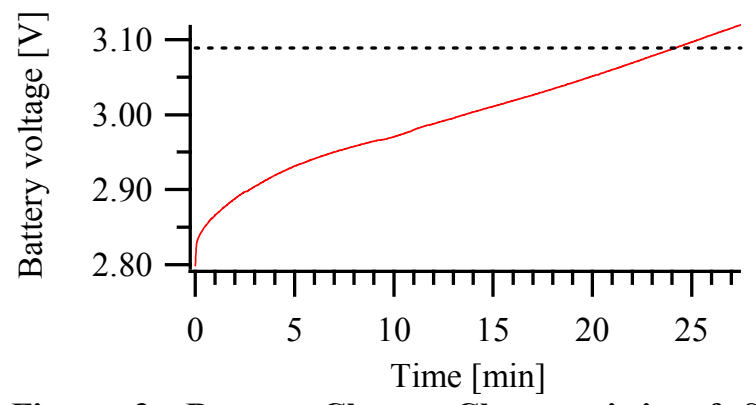

Figure 3: Battery Charge Characteristic of Seiko MS920SE (11mAh) - Dotted Line: possible threshold Voltage for estimating a full battery. The input voltage of the BQ25504 was $590 \mathrm{mV}$ at a series resistor of 47.126 $\Omega$ )

\subsection{Source of energy}

As a replacement for an energy harvesting device we use the DC power supply E3631A (Agilent Technologies). To limit the amount of energy provided by the power supply a series resistor is used. The voltage is measured behind the series resistor, so the input current and input power can be estimated.

\section{Results}

The following figure shows the characteristic of the BQ25504 when reaching the overvoltage of a battery.

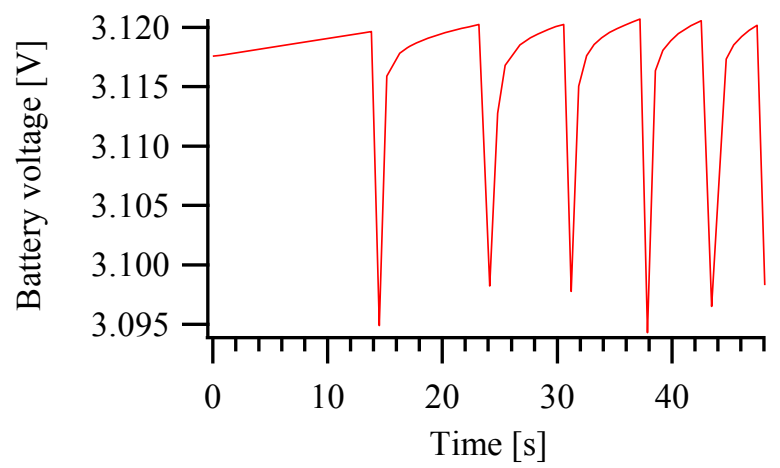

Figure 4: On-Off Characteristic of BQ25504 - Overvoltage is set so $3.12 \mathrm{~V}$ 
When the battery voltage reaches the programmed overvoltage, the BQ25504 stops charging the battery until the voltage decreases by $18-89 \mathrm{mV}$ and then restarts charging [5]. This can also be seen at the decreasing input power (see Figure 7).

\subsection{Energy Consumption}

The following table gives an overview of the energy consumption required by the components of the experimental setup.

Table 2: Power Consumption at 3.1 V

\begin{tabular}{|l|c|c|c|}
\hline \multicolumn{1}{|c|}{ Device } & Power & $\begin{array}{c}\text { On/Off- } \\
\text { Time }\end{array}$ & $\begin{array}{c}\text { Energy Dissi- } \\
\text { pation per On- } \\
\text { Time }\end{array}$ \\
\hline BQ25504 & $3.1 \mu \mathrm{W}$ & $100 \%$ & --- \\
\hline MSP430 & $1.5 \mu \mathrm{W}$ & $100 \%$ & --- \\
\hline $\begin{array}{l}\text { Comparator } \\
\text { with Refer- } \\
\text { ence }\end{array}$ & $3.6 \mu \mathrm{W}$ & $\begin{array}{c}12 \mathrm{~ms} / \\
30 \mathrm{~s}\end{array}$ & $43.2 \mathrm{~nJ}$ \\
\hline $\begin{array}{l}\text { FM Trans- } \\
\text { mitter }\end{array}$ & $6.5 \mathrm{~mW}$ & $\begin{array}{c}2.5 \mathrm{~ms} / \\
---{ }^{1}\end{array}$ & $16.3 \mu \mathrm{J}$ \\
\hline
\end{tabular}

The power consumption of the BQ25504 was taken from the Datasheet [5] whereas the other values were measured using the Digital Multimeter 34401A (Agilent) at an input voltage of $3.1 \mathrm{~V}$.

\subsection{Threshold power}

Due to the low efficiency of the BQ25504 the minimum threshold input power, for beginning charging the battery, was determined. The efficiency of the BQ25504 depends on the input current and voltage. With a given series resistance $(370.426 \Omega)$ the input voltage was decreased until the battery drained power. Our results show a need of 415 $\mu \mathrm{W}$ to charge the battery.

\subsection{Long-Term Tests}

A first long-term test was realized with no further device activated, but the BQ25504 for charging the $\mathrm{CBC} 012$ battery. In the beginning the input power was set to about $380 \mu \mathrm{W}$. The series resistor was $370.426 \Omega$ at an input voltage of $780 \mathrm{mV}$.

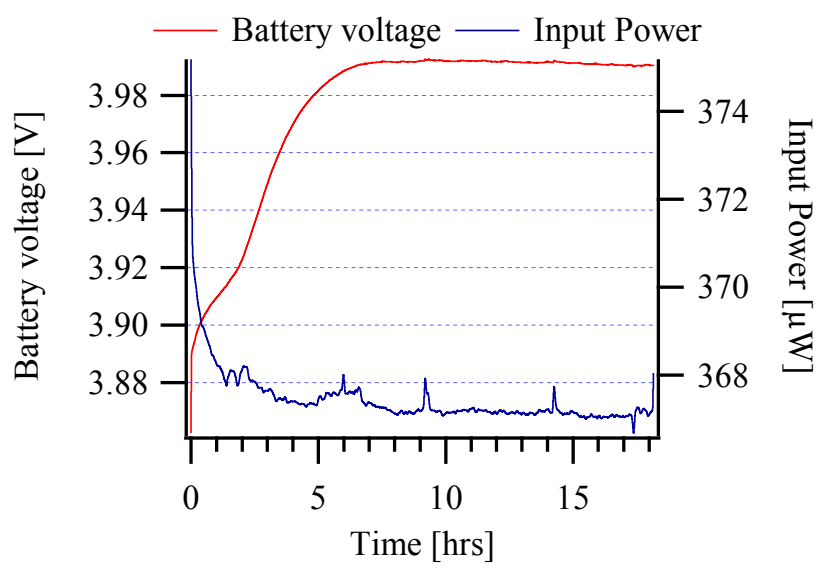

Figure 5: Charging curve - The BQ25504 is not able to fully charge the battery. At the end the battery voltage is decreasing.

The results (see Figure 5) show that after a time of approximately 8 hours, the voltage of the battery doesn't increase but rather decreases slowly. Also the input power is slightly decreasing and the BQ25504 is taking energy from the source but cannot charge the battery with it.

In another long-term test the module was tested at normal state. The input power was set to about $415 \mu \mathrm{W}$, the series resistor was $370.426 \Omega$ at an input voltage of $830 \mathrm{mV}$. The battery CBC012 was used.

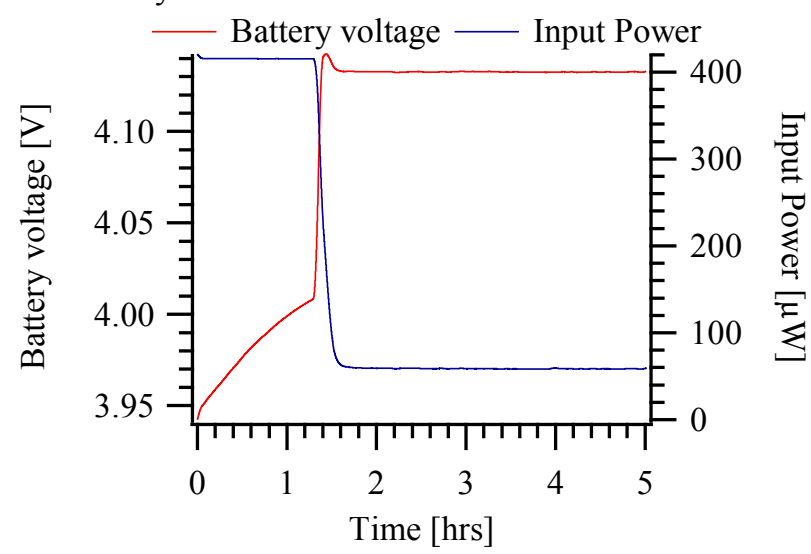

Figure 6: Charging Curve - The input power is large enough to fully charge the battery and send data wirelessly.

The following figure shows an excerpt of the voltage and input power when the threshold voltage is reached and data is transmitted via the VCO.

\footnotetext{
${ }^{1}$ Depends on state of charge of battery
} 


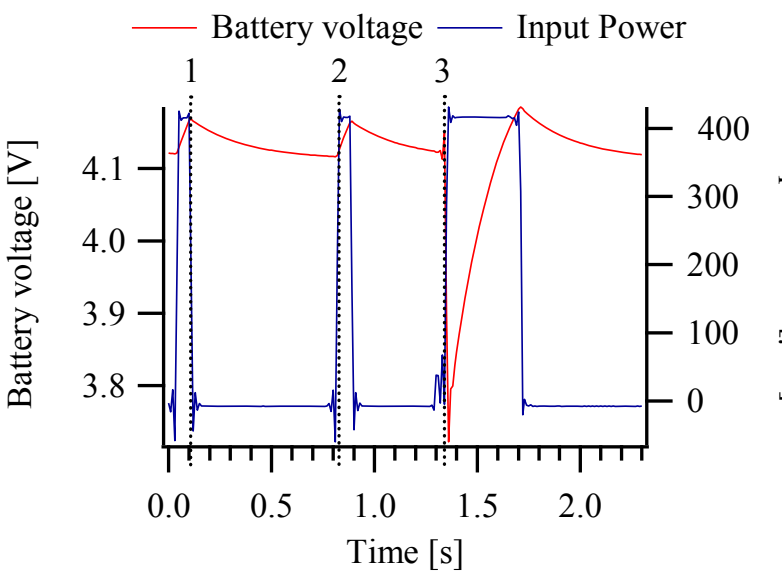

Figure 7: Excerpt of the voltage and power curve - 1 - Boost converter is turned off, Battery overvoltage reached; 2 - Boost converter is turned on, charging Battery; 3 - Comparator is turned on, Voltage exceeds threshold voltage, FM module is activated and data is transmitted.

When the VCO is active, the voltage drops down by 300 $\mathrm{mV}$ because of the large current needed for the device. For this short time, the battery was able to give the amount of power.

The next figure shows the battery voltage after turning off the power source when the battery was fully charged.

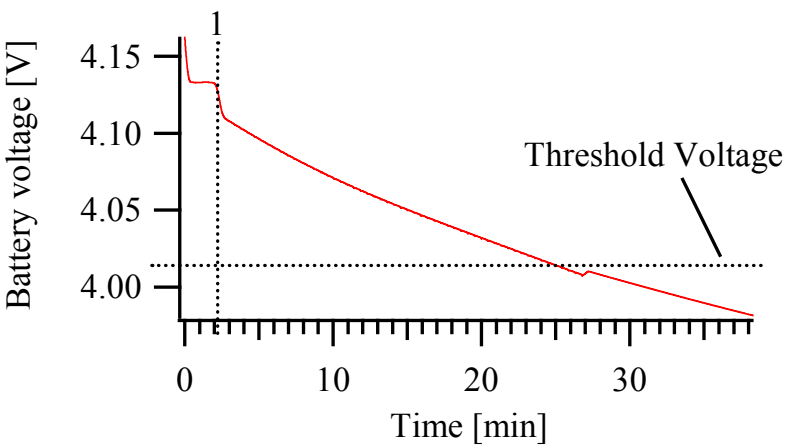

Figure 8: Battery voltage after switching off the power source. 1 - Time of switching off power source

The device was able to send 54 messages (interval of $30 \mathrm{~s}$ ) until the threshold voltage was reached. After dropping below the threshold voltage, the device stopped sending data.

\section{Conclusion}

In order to implement a biosensor in human bodies a discrete circuitry, as used in this work, is too bulky. Highly integrated Micro-Electro-Mechanical Systems (MEMS) are state of the art entailing also lower power consumption and are more convenient for implanting in human bodies.

Nevertheless we built a power management system to store the little amount of energy provided by a power source of $415 \mu \mathrm{W}$.

In further studies real energy harvesting devices should be used for testing purposes. With a specific energy harvester, the boost converter could be improved to meet better efficiency. For real piezoelectric motion harvesters, the minimum input power of our power management should be divided by more than four [8]. Optimizing the Maximum Power Point Tracking, which can be configured to meet the harvesters' characteristics, would be a good point to continue with.

\section{$5 \quad$ References}

[1] Raghunathan, V.; Chou, P. H.: Design and Power Management of Energy Harvesting Embedded Systems: ISLPED'06 Proceedings of the 2006 International Symposium on Low Power Electronics and Design 2006.

[2] Berndt, D.: Maintenance free batteries. Lead acid, nickel - cadmium, nickel - metal hydride ; a handbook of battery technology, 2. ed., reprinted. Taunton 1997.

[3] Vincent, C. A.; Scrosati, B.: Modern batteries. An introduction to electrochemical power sources, 2nd. London ;, Baltimore, Md., USA 1997.

[4] Retzbach, L.: Akkus und Ladegeräte, 13., überarb. Villingen-Schwenningen 2002.

[5] Texas Instruments: Ultra Low Power Boost Converter with Battery Management for Energy Harvester Applications.

URL: http://www.ti.com/lit/ds/symlink/bq25504.p df.

[6] Texas Instruments: MIXED SIGNAL MICROCONTROLLER - G2553.

URL: http://www.ti.com/lit/ds/symlink/msp430g2 553.pdf.

[7] MAXIM: 3-Pin, Ultra-Low-Power SC70/SOT23 Voltage Detectors - MAX6376.

URL: http://datasheets.maximic.com/en/ds/MAX6375-MAX6380.pdf.

[8] Mitcheson, P.; Yeatman, E.; Rao, G.; Holmes, A.; Green, T.: Energy Harvesting From Human and Machine Motion for Wireless Electronic Devices. In: Proceedings of the IEEE 96 (2008) 9, S. 1457 86. 